\title{
A study of microbial spectra of soils potentially relevant for the formation of stable soil foci of anthrax
}

\author{
A.V. Kalinin*, O.I. Tsygankova, E.A. Koteneva, A.V. Abramovich, G.A. Pechkovsky \\ Stavropol Antiplague Institute of the Rospotrebnadzor, Stavropol, Russia \\ *e-mail:jugask@mail.ru
}

Key words: bacilli, Bacillus anthracis, phenotypic variability, heterogeneity of population, pathogenic properties, morphofunctional forms

Motivation and Aim: The members of the genus Bacillus constitute a group of widespread microorganisms which are being isolated from the soil. This heterogeneous taxonomic group of spore-forming bacteria contains more than 65 species with new species being discovered constantly [1]. Under favorable conditions of the environment the physiological and metabolic universality of bacilli ensures fast germination of spores [2]. Basically, the genus Bacillus consists of saprophytic organisms, however the greatest interest of medical microbiology is focused on the taxonomic group Bacillus cereus (sensu lato), since it comprises three species causing diseases in mammals: Bacillus anthracis, Bacillus cereus and Bacillus thuringiensis. B. anthracis is the etiological agent of anthrax. Many mammals and some birds are sensitive to this agent, but basically this species affects herbivores. After the death of the infected animal the process of spore formation begins in organic remains of the dead animal in the presence of oxygen. The soil in the places of death and burial of animals serves as a primary source for further infections during many tens of years. An assumption has been made that in "the areas of incubation" in soils rich with mineral and organic substances with $\mathrm{pH}$ above 6.0 and temperature above $15^{\circ} \mathrm{C}$ which topographically correlate with the location of areas in which infections of animals with $B$. anthracis take place from time to time, $B$. anthracis can not only persist during the interepizootic period, but also propagate [1]. It is known that $B$. cereus can exist in the root zone of plants, and it is necessary to take into account the probability of germination of $B$. anthracis spores in the rhizosphere and propagation of vegetative cells of $B$. anthracis in this ecological niche. It has been confirmed on modelling organisms that viable bacilli of anthrax can exist in the rhizosphere [2]. Recent ecological studies have shown that anthrax bacilli can interact with earthworms, soil amoebas and bacteriophages. It has been revealed that the influence of bacteriophages on $B$. anthracis can cause phenotypic changes in bacilli; these changes make them capable of endosymbiont existence in the organisms of earthworms and of functioning as saprophytes in the soil and water. Hence, it may be assumed that amoebas and probably other soil protozoa can serve as potential host organisms for anthrax bacilli, and the life cycle of this bacteria is not limited by the standard paradigm of the development of anthrax. The major factors of counteraction to protective mechanisms of the macro organism during the infectious phase - the polyglutamic capsule and exotoxins - cannot ensure persistence and viability of the culture which comes into the environment. At this stage strains capable of forming the high-grade spores, highly resistant to adverse physical, chemical and biological factors of the environment, more often of soil, gain advantages.

Conclusion: Inhomogeneity of populations of strains of the causative agent of anthrax with respect to many properties in the absence of selective factors operating in organisms of sensitive animals may lead to the appearance and accumulation of strain subcultures which lost pathogenic properties or which possess steady complexes of atypical phenotypic and genetic properties. The estimation of roles of various soil foci of anthrax in preservation of the reservoir of the infection may be carried out taking into account studies of population changes in B. anthracis strains in case of their long persistence in soil and preservation of the potential of pathogenicity of such "soil" populations.

\section{References}

1. Hugh-Jones M. (2009) The ecology of Bacillus anthracis. J. Mol Aspects Med. 30(6):356-67.

2. Mullins J.C. et al. (2013) Ecological niche modeling of Bacillus anthracis on three continents: Evidence for genetic-ecological divergence? PLoS ONE. 8(8):e72451.

3. Bazinet A.L. (2017) Pan-genome and phylogeny of Bacillus cereus sensu lato. BMC Evol Biol. 17:176. 